\title{
Efecto combinado de cepa de levadura y Terroir en vinos Malbec de Mendoza
}

\section{Combined effect of yeast strains and Terroir on Malbec wines from Mendoza}

\author{
Dolores Pérez ${ }^{1}$, Carolina Capaldi ${ }^{2}$, Laura Mercado $^{3}$, Adriana Malizia $^{4}$ y Santiago Sari ${ }^{1}$ \\ ${ }^{1}$ Centro de Estudios de Enología, Estación Experimental Agropecuaria Mendoza, Instituto Nacional de Tecnología \\ Agropecuaria (INTA), San Martín 3853, \\ 5507 Luján de Cuyo, Mendoza, Argentina. \\ ${ }^{2}$ Facultad de Ciencias Agrarias, Universidad Nacional de Cuyo, Almte. Brown 500, 5507, Luján de Cuyo, Mendoza, \\ Argentina \\ ${ }^{3}$ Laboratorio de Microbiología Enológica Estación Experimental Agropecuaria Mendoza, Instituto Nacional de \\ Tecnología Agropecuaria (INTA), San Martín 3853, 5507 Luján de Cuyo, Mendoza, Argentina. \\ ${ }^{4}$ LALLFERM S.A., Lateral Este de Acc. Sur 1751, Luján de Cuyo, Mendoza, Argentina.
}

\begin{abstract}
Resumen. Las cepas de levaduras producen atributos distintivos en los vinos, como así también, la uva proveniente de diferentes zonas puede modificar el flavour del vino. El objetivo del presente trabajo fue evaluar el efecto producido por levaduras comerciales (EC1118, Tango Malbec, Flavia e Ionys) e indígenas sobre vinos Malbec provenientes de Ugarteche, El Cepillo y Gualtallary (Mendoza, Argentina). Se determinaron perfiles fermentativos, controles de implantación, parámetros físico-químicos y cromáticos generales y análisis sensorial de los vinos. Se aplicó ANAVA de dos vías con test LSD Fisher sobre los parámetros y se graficó un ACP. Las levaduras comerciales se implantaron en un 100\%, mientras que los perfiles genéticos de las fermentaciones espontáneas fueron diferentes entre sí. El factor región, evidenció un importante efecto, destacándose los vinos de Ugarteche con altos niveles de $\mathrm{pH}$ y bajos valores de alcohol, intensidad colorante, IPT y antoncianos totales. El factor levadura reveló efectos significativos respecto a la levadura Ionys, la cual en las tres regiones, finalizó su fermentación dos días después que el resto de las levaduras y en sus vinos se destacó la nota a fruta fresca y la baja intensidad de astringencia y amargo. Sumado a esto, produjo $0.5 \%$ menos grados de alcohol, 0.2 menos de $\mathrm{pH}$.
\end{abstract}

\begin{abstract}
Yeast strains may impact on wine characteristics, producing distinct attributes. In addition, it is generally accepted, that grape must from different agro-ecological zones can impart on wine flavour. The aim of the present research was to evaluate the effects of commercial (EC1118, Tango Malbec, Flavia and Ionys) and indigenous yeast strains on chemical Malbec wines made from Ugarteche, El Cepillo and Gualtallary vineyards (Mendoza, Argentina). Fermentation profile, implantation controls, general chemical and chromatic wine parameters and sensory characterization were determined. Two-way ANOVA with LSD Fisher test and PCA were applied. In all commercial inoculated fermentations, implantation showed almost $100 \%$ of similarity, while spontaneous fermentations exhibited different profiles. The region had a significant individual effect, where wines from Ugarteche region were characterized by lower alcohol degree, spectrophotometric detection at $280 \mathrm{~nm}$, colour intensity and total anthocyanidins and huge $\mathrm{pH}$ values, in wines. The yeast factor presented significant effects mainly due to wines fermented by Ionys yeast strain, which its fermentation duration lasted two more days than the others yeast, but it produced outstanding effects on sensorial aspects of wines, favouring fresh-fruit notes and lowering bitterness and astringency. Furthermore, this yeast produced a reducing effect on $\mathrm{pH}$ and alcohol degree of about 0.2 points and $0.5 \%$, respectively.
\end{abstract}

\section{Introducción}

El cultivar Malbec (Vitis vinifera L), oriundo de Burdeos-Francia es considerado una variedad emblemática de la producción vitivinícola argentina; ya que permite lograr excelentes vinos apreciados nacional e internacionalmente. En la actualidad existen 40.401 ha de esta variedad en Argentina, de las cuales el 85,8\% se encuentra en Mendoza [1]. La Zona Alta del Río
Mendoza es la región vitícola más tradicional en el cultivo de este cepaje, ubicada en el pedemonte de la Cordillera de Los Andes, a $33^{\circ}$ de latitud sur. Organolépticamente el Malbec se caracteriza por una alta intensidad colorante, con matices violetas y negros mientras que a nivel aromático, se pueden apreciar notas a ciruelas pasas, moras, violetas y anís como también algunas notas herbáceas [2]. 
La composición fenólica en las variedades de uva tinta es un parámetro cada vez más importante en la calidad del vino. Los compuestos fenólicos se clasifican en diferentes grupos, entre los cuales los antocianos y los flavonoides son los que tienen mayor influencia sobre el color, su evolución y otras características organolépticas relacionadas con su calidad [3].

Existen diversos factores que influyen sobre la acumulación de compuestos fenólicos en la uva y por lo tanto en el vino, uno de ellos es el factor edafoclimático. Factores ambientales como la luz y la temperatura, que caracterizan diferentes zonas de cultivo, inciden en forma directa en la actividad fotosintética de la planta y sobre las enzimas de la ruta fenilpropanoide modificando la biosíntesis de estos compuestos [4-6].

El término francés "Terroir" se refiere a la relación entre un determinado vino y una zona específica de producción del mismo, la cual le provee características y atributos únicos con respecto a otras zonas [7]. Dentro de este concepto, también se encuentra la microbiota nativa, la cual se ha demostrado que puede ser característica en una determinada zona vitícola [8].

Por otro lado, el pH, ya sea en el mosto o en el vino, es otro aspecto importante a tener en cuenta en relación al efecto sobre los antocianos. Estos pigmentos principales responsables del color de los vinos se encuentran en forma libre $\mathrm{y}$ en equilibrio constante entre diferentes formas químicas en función del $\mathrm{pH}$, intensificándose las tonalidades rojas y aumentando el color total cuando los valores de $\mathrm{pH}$ son bajos [3]. Sumado a esto, la influencia de las levaduras que intervienen en la fermentación de los mostos sobre el color final del vino, es uno de los factores que ha sido estudiado más recientemente. Algunas cepas de levaduras debido a una desviación en su ruta metabólica durante la fermentación alcohólica, pueden producir un efecto acidificante sobre el mosto en fermentación [9, 10] produciendo sobre el color del vino el efecto arriba mencionado. Además, los bajos $\mathrm{pH}$ favorecen el fenómeno de copigmentación, típico en vinos jóvenes, en el cuál a través de uniones no covalentes tipo "sándwich" entre antocianos y copigmentos se favorece la estabilidad del color en el tiempo como también se produce una intensificación del mismo [11]. Sin embargo, se ha postulado que las levaduras vínicas también pueden actuar eliminando antocianos, adsorbiéndolos sobre su pared y también hidrolizando los heterósidos de antocianos por la actividad $\beta$ glucosidasa, dando lugar a agliconas más inestables [3]. En resumen, según la cepa de levadura utilizada pueden variar tanto el color del vino como la concentración de antocianos, por lo que, la influencia sobre el color es uno de los criterios primordiales en la selección de levaduras para la vinificación en tinto.

El aroma es otro importante parámetro de calidad en el vino. Debido a que es el resultado de diversos procesos biológicos, químicos y tecnológicos que ocurren desde la uva hasta el vino, se lo clasifica según su origen en: aroma primario o varietal, secundario o fermentativo y terciario o de crianza [12]. El aroma de origen varietal está constituido por sustancias volátiles (aromáticas) y no volátiles (no aromáticas) generadas durante el desarrollo de la baya y que contribuyen a la diferenciación varietal. Por lo tanto, dependen de las rutas metabólicas de la vid y consecuentemente están mayormente influenciados por factores que afecten a la planta [13]. Sin embargo, los procesos tecnológicos y biológicos aplicados durante la obtención del vino también pueden influenciar su contenido, por ejemplo, ciertas levaduras hidrolizan las formas ligadas de estos compuestos y los transforman en aromáticos [14], por otro lado, se ha determinado que las bajas temperaturas de fermentación favorecen la retención de estos aromas [15].

Los compuestos aromáticos de origen fermentativo provienen de dos procesos biológicos, la fermentación alcohólica, llevada a cabo principalmente por levaduras del género Saccharomyces cerevisiae y la fermentación maloláctica, llevada a cabo por bacterias lácticas. Es el grupo aromático más abundante y es el responsable, entre otras, de la nota vinosa. Su composición y concentración dependen de factores que impactan sobre el metabolismo de los microorganismos implicados en el proceso fermentativo, tales como temperatura de fermentación, nutrición, cepa de levadura, composición del mosto, etc.[15]. Se ha demostrado también, que existen diferencias en la producción de metabolitos secundarios entre cepas de levaduras, lo que influye en las características químicas y organolépticas del vino $[9$, 16].

A partir de lo anteriormente expuesto, resulta fundamental el contribuir al conocimiento del efecto individual y de interacción entre levaduras y zonas vitícolas de Mendoza sobre los parámetros relacionados con la calidad de los vinos Malbec.

\section{Materiales y métodos 2.1. Materia Prima}

Se utilizaron uvas de viñedos pertenecientes a la bodega Doña Paula ubicados en tres zonas diferentes de la provincia de Mendoza, cuyas características se detallan en la siguiente Tabla 1.

\subsection{Microorganismos}

Para llevar a cabo la fermentación alcohólica se utilizaron las siguientes levaduras secas activas provistas por la empresa Lalferm S.A.: Lalvin EC1118 (Saccharomyces cerevisiae bayanus), Ionys (Saccharomyces cerevisiae), Tango Malbec (Saccharomyces cerevisiae) y Flavia (Metschnikowia pulcherrima). Los tratamientos con Flavia, 24 horas después de su inoculación, fueron secuencialmente implantados con EC1118. Paralelamente, como testigo, se realizaron fermentaciones espontaneas a partir de la flora nativa contenida en la uva de cada zona. 
Tabla 1. Características agro-ecológicas de los viñedos.

\begin{tabular}{lccccccc}
\hline \multicolumn{1}{c}{ Localidad } & $\begin{array}{c}\text { Sistema de } \\
\text { Conducción }\end{array}$ & Latitud & Longitud & $\begin{array}{c}\text { Altitud } \\
(\mathbf{m})\end{array}$ & Suelo & $\begin{array}{c}\text { Riego } \\
\text { Rendimiento } \\
(\mathbf{K g} / \mathbf{h a})\end{array}$ \\
\hline Ugarteche, Luján de Cuyo & Espaldero & $33^{\circ} 15^{\prime} 00^{\prime \prime}$ & $68^{\circ} 57^{\prime} 38^{\prime \prime}$ & 1012 & Franco-Arenoso & Goteo & 8000 \\
El Cepillo, San Carlos & Espaldero & $33^{\circ} 49^{\prime} 13,6^{\prime \prime}$ & $69^{\circ} 11^{\prime} 51,7^{\prime \prime}$ & 1117 & Franco-Arenoso & Goteo & 6500 \\
Gualtallary, Tupungato & Espaldero & $33^{\circ} 23^{\prime} 13,07^{\prime \prime}$ & $69^{\circ} 13^{\prime} 52^{\prime \prime}$ & 1293 & Franco-Arenoso & Goteo & 6000 \\
\hline
\end{tabular}

\subsection{Protocolo de vinificación}

Por cada región, se descobajaron y molieron $20 \mathrm{~kg}$ de uva colocados en recipientes plásticos de $25 \mathrm{~L}$ de capacidad, esto se realizó por triplicado. Se analizaron los parámetros básicos de rutina en todos los mostos ( ${ }^{\circ} \mathrm{Brix}, \mathrm{pH}$, acidez total) y luego fueron adicionados con solución acuosa de $3 \mathrm{~g} / \mathrm{hL}$ de metabisulfito de potasio y de ser necesario, se corrigió la acidez a $6 \mathrm{~g} / \mathrm{L}$ con ácido tartárico.

Se aplicó un diseño multifactorial con dos factores (Zonas y Levaduras), con 3 niveles para el primer factor y 5 niveles para el segundo factor, basados en la cantidad de zonas y las levaduras utilizadas.

La hidratación y aclimatación de las levaduras comerciales se realizó según protocolo brindado por el fabricante. Las fermentaciones se llevaron a cabo a una temperatura controlada de $25^{\circ} \mathrm{C}$ y el seguimiento diario de las mismas se realizó a través del control de la densidad del mosto $\left(\mathrm{g} / \mathrm{cm}^{3}\right)$ con un periodo de maceración para todos igual correspondiente a 10 días. La nutrición se aplicó en dos momentos, al principio de la FA y al llegar a una densidad igual a $1,070 \mathrm{~g} / \mathrm{cm}^{3}$.

Finalizando la fermentación alcohólica se tomó una muestra de cada repetición para llevar a cabo un control de implantación de las mismas, seguidamente, se inocularon bacterias Oenococcus oeni Lalvin VP41 (Lalferm S.A). Finalizado el período de maceración se realizó el descube de los tratamientos continuando con la fermentación maloláctica $\left(20{ }^{\circ} \mathrm{C}\right)$. Una vez finalizados los procesos fermentativos, se adicionó anhídrido sulfuroso hasta $35 \mathrm{mg} / \mathrm{L}$ y se procedió con las estabilizaciones pertinentes a una temperatura aproximada de $4-8{ }^{\circ} \mathrm{C}$, durante 2 meses. El envasado se realizó en botellas de vidrio de $750 \mathrm{~mL}$ de capacidad con tapa a rosca, previo trasiego.

\subsection{Determinaciones analíticas}

Las siguientes determinaciones analíticas se realizaron en mosto y vino según protocolos de la Organización Internacional de la Viña y el Vino [17, 18]: sólidos solubles por refractrometría, acidez total, acidez volátil, $\mathrm{pH}$, azúcares reductores, grado alcohólico por método aerométrico. Los parámetros relacionados con el color se determinaron siguiendo lo descripto por Zamora, 2003: índice de polifenoles totales a 280nm, antocianos totales, intensidad colorante. El control de implantación de las cepas de levaduras se determinó mediante la técnica de diferenciación intraespecífica, PCR Interdelta (Lallemand, Canadá). La caracterización sensorial se realizó mediante un análisis descriptivo no estructurado mediante panel entrenado ( 15 personas) perteneciente al Centro de Estudios en Enología, EEA Mendoza INTA.

\subsection{Tratamiento de datos y análisis estadístico}

Primeramente se verificó la normalidad de los datos, procesados por el software estadístico Infostat $\AA$. Se aplicó análisis de la varianza multifactorial y Test LSD Fisher para obtener una comparación de las medias buscando diferencias significativas entre los diferentes tratamientos y evaluar el efecto de los factores con un nivel de significancia de $\mathrm{p} \leq 0,05$. Además, se les aplicó un análisis multivariado, utilizando como método de reducción de información, el Análisis de Componentes Principales (ACP).

Tabla 2. Datos iniciales de los mostos de las diferentes zonas vitícolas

\begin{tabular}{|c|c|c|c|}
\hline Variable * & El Cepillo & Gualtallary & Ugarteche \\
\hline${ }^{\circ}$ Brix & $23,4 \pm 0,26^{\mathrm{a}}$ & $24,65 \pm 0,56^{\mathrm{c}}$ & $24,3 \pm 0,1^{\mathrm{b}}$ \\
\hline $\mathrm{pH}$ & $3,52 \pm 0,02^{\mathrm{b}}$ & $3,66 \pm 0,02^{\mathrm{c}}$ & $3,42 \pm 0,02^{\mathrm{a}}$ \\
\hline Acidez Total & $5,25 \pm 0,36^{\mathrm{b}}$ & $4,35 \pm 0,17^{\mathrm{a}}$ & $5,86 \pm 0,1^{\mathrm{c}}$ \\
\hline
\end{tabular}

\section{Resultados y discusión}

Con respecto a los datos iniciales de los mostos de uva Malbec pertenecientes a cada zona (Tabla 2) se pudo observar que se diferenciaron ampliamente mostrando una marcada influencia de la zona de origen sobre la uva. Gualtallary alcanzó un mayor grado de madurez y mayor nivel de $\mathrm{pH}$ con un consecuente nivel de acidez total inferior al resto. En cuanto a las características de El Cepillo, se destacó su menor nivel azucarino pero niveles intermedios de $\mathrm{pH}$ y acidez respecto al resto, siendo Ugarteche el mosto con mayores niveles de acidez y $\mathrm{pH}$ pero de madurez intermedia. Este último resultado llama la atención si se considera que este viñedo se encuentra en una zona de menor altura y más cálida entre las estudiadas en este trabajo, por lo que se esperaban mostos con menor acidez [5]. Sin embargo, a pesar de los resultados del análisis estadístico, estas diferencias entre zonas pueden ser consideradas de poca magnitud e impacto a nivel enológico. 
Tabla 3. Duración promedio de las fermentaciones y valores promedios \pm desviación estándar de los análisis finales de los vinos obtenidos de cada región geográfica y levadura.

\begin{tabular}{|c|c|c|c|c|c|c|}
\hline Zona & Levaduras & Duración de la FA (días) & $\mathrm{pH}$ & Ac.Total (g/L) & Ac.Volátil (g/L) & Alcohol $(\% \mathrm{v} / \mathrm{v})$ \\
\hline \multirow[t]{4}{*}{ El Cepillo } & EC1118 & 8 & $3,72 \pm 0,01^{\mathrm{bcd}}$ & $4,84 \pm 0,05^{\mathrm{cd}}$ & $0,33 \pm 0,07^{\text {bcd }}$ & $13,95 \pm 0,00^{\mathrm{e}}$ \\
\hline & Flavia & 8 & $3,68 \pm 0,01 \mathrm{bc}$ & $4,48 \pm 0,56^{a b c}$ & $0,25 \pm 0,00^{a b c}$ & $13,73 \pm 0,18^{\mathrm{cd}}$ \\
\hline & Tango Malbec & 8 & $3,73 \pm 0,01^{\text {cde }}$ & $4,54 \pm 0,05^{\mathrm{bcd}}$ & $0,35 \pm 0,10^{\text {cde }}$ & $13,9 \pm 0,00^{\mathrm{de}}$ \\
\hline & Ionys & 10 & $3,59 \pm 0,02^{\mathrm{a}}$ & $5,55 \pm 0,21^{\mathrm{e}}$ & $0,18 \pm 0,02^{\mathrm{a}}$ & $13,4 \pm 0,07^{\mathrm{ab}}$ \\
\hline \multirow[t]{4}{*}{ Gualtallary } & EC1118 & 8 & $3,86 \pm 0,02^{g}$ & $4,50 \pm 0,11^{\mathrm{abc}}$ & $0,34 \pm 0,03^{\mathrm{bcd}}$ & $14,83 \pm 0,11^{\mathrm{g}}$ \\
\hline & Flavia & 9 & $3,76 \pm 0,04$ def & $4,87 \pm 0,00^{\mathrm{cd}}$ & $0,35 \pm 0,04^{\text {cde }}$ & $14,78 \pm 0,04^{\mathrm{g}}$ \\
\hline & Tango Malbec & 8 & $3,80 \pm 0,01^{\text {efg }}$ & $4,76 \pm 0,37^{\mathrm{cd}}$ & $0,45 \pm 0,06^{\mathrm{e}}$ & $14,75 \pm 0,00^{g}$ \\
\hline & Ionys & 11 & $3,65 \pm 0,01 \mathrm{ab}$ & $5,55 \pm 0,21^{\mathrm{e}}$ & $0,24 \pm 0,05^{\mathrm{ab}}$ & $14,23 \pm 0,11^{\mathrm{f}}$ \\
\hline \multirow[t]{7}{*}{ Ugarteche } & EC1118 & 8 & $4,02 \pm 0,03^{\mathrm{hi}}$ & $4,05 \pm 0,00^{\mathrm{a}}$ & $0,40 \pm 0,00^{\mathrm{de}}$ & $14,00 \pm 0,07^{\mathrm{e}}$ \\
\hline & Flavia & 8 & $3,97 \pm 0,04^{h}$ & $4,47 \pm 0,05^{a b c}$ & $0,25 \pm 0,03^{a b c}$ & $13,58 \pm 0,04^{\mathrm{bc}}$ \\
\hline & Tango Malbec & 7 & $4,07 \pm 0,04^{\mathrm{i}}$ & $4,13 \pm 0,11^{\mathrm{ab}}$ & $0,28 \pm 0,07^{\mathrm{abc}}$ & $13,98 \pm 0,11^{\mathrm{e}}$ \\
\hline & Ionys & 11 & $3,83 \pm 0,07^{\mathrm{fg}}$ & $5,00 \pm 0,04^{\mathrm{d}}$ & $0,18 \pm 0,02^{\mathrm{a}}$ & $13,38 \pm 0,04^{\mathrm{a}}$ \\
\hline & Zona $(\mathrm{Z})$ & & $* * *$ & $* *$ & $* *$ & $* * *$ \\
\hline & Levadura (L) & & $* * *$ & $* * *$ & $* *$ & $* * *$ \\
\hline & $Z \times L$ & & $*$ & $*$ & $*$ & $*$ \\
\hline
\end{tabular}

Letras diferentes indican diferencias estadísticas de la aplicación del análisis de la varianza con test LSD Fisher, $\mathrm{p}$-valor $<0,05$. $* * * \mathrm{p}<0,001 ; * * \mathrm{p}<0,05$; $* \mathrm{p}>0,05$.

El control de implantación reveló que en cada tratamiento las fermentaciones se realizaron exitosamente con las cepas inoculadas, resultando un nivel de implantación cercano al $100 \%$ en todos los casos (datos no mostrados). Con respecto a las fermentaciones espontáneas se observaron diferentes perfiles genéticos demostrando que las cepas nativas difieren entre y dentro de una misma zona [19] y que en ningún caso estos perfiles coincidieron con las cepas comerciales. A partir de este resultado se decidió no tomar en cuenta los tratamientos de fermentaciones espontáneas en el análisis factorial debido a que el factor levadura no era el mismo entre repeticiones y zonas.

Tabla 4. Intensidad colorante (IC), índice de polifenoles totales a $280 \mathrm{~nm}$ (IPT) y antocianos totales (AT) de los vinos de tres zonas geográficas fermentados por diferentes levadaduras.

\begin{tabular}{|c|c|c|c|c|}
\hline Zona & Levaduras & IC & IPT & $\mathrm{AT}(\mathrm{mg} / \mathrm{L})$ \\
\hline \multirow[t]{4}{*}{ El Cepillo } & EC1118 & $1,44 \pm 0,04^{a b}$ & $86,85 \pm 1,63^{d}$ & $918,09 \pm 32,48^{b c}$ \\
\hline & Flavia & $1,65 \pm 0,15^{\mathrm{cd}}$ & $85,2 \pm 13,86^{\mathrm{d}}$ & $985,69 \pm 64,97^{\text {cde }}$ \\
\hline & Tango Malbec & $1,85 \pm 0,12^{\mathrm{e}}$ & $94,3 \pm 5,94^{\mathrm{d}}$ & $1051,09 \pm 45,48^{\text {def }}$ \\
\hline & Ionys & $1,99 \pm 0,03{ }^{\text {ef }}$ & $83,9 \pm 7,78^{\mathrm{cd}}$ & $924,66 \pm 31,86^{\mathrm{bcd}}$ \\
\hline \multirow[t]{4}{*}{ Gualtallary } & EC1118 & $1,58 \pm 0,11^{b c}$ & $112,6 \pm 0,57^{\mathrm{e}}$ & $1052,19 \pm 9,28^{\text {ef }}$ \\
\hline & Flavia & $1,65 \pm 0,03^{\mathrm{cd}}$ & $92,85 \pm 9,4^{\mathrm{d}}$ & $1170,75 \pm 37,12^{\mathrm{f}}$ \\
\hline & Tango Malbec & $1,83 \pm 0,08^{\mathrm{de}}$ & $113,65 \pm 10,11^{\mathrm{e}}$ & $1173,59 \pm 20,73^{\mathrm{f}}$ \\
\hline & Ionys & $2,1 \pm 0,08^{\mathrm{f}}$ & $82,26 \pm 5,06^{\text {bcd }}$ & $1029 \pm 158,39^{\text {cde }}$ \\
\hline \multirow[t]{7}{*}{ Ugarteche } & EC1118 & $1,43 \pm 0,07^{\mathrm{ab}}$ & $69,35 \pm 5,87^{\mathrm{abc}}$ & $833,74 \pm 8,85^{\mathrm{ab}}$ \\
\hline & Flavia & $1,37 \pm 0,12^{\mathrm{a}}$ & $55,65 \pm 0,49^{\mathrm{a}}$ & $903 \pm 63,73^{b c}$ \\
\hline & Tango Malbec & $1,47 \pm 0,09^{a b c}$ & $68,05 \pm 6,15^{\mathrm{ab}}$ & $913,72 \pm 33,72^{b c}$ \\
\hline & Ionys & $1,5 \pm 0,08^{a b c}$ & $54,6 \pm 5,09^{a}$ & $764,97 \pm 16,4^{a}$ \\
\hline & Zona (Z) & $* * *$ & $* * *$ & $* * *$ \\
\hline & Levadura (L) & $* * *$ & $*$ & $* *$ \\
\hline & $\mathrm{Z} \times \mathrm{L}$ & $* *$ & $* *$ & $*$ \\
\hline
\end{tabular}

Valores promedios \pm desviación estandar, letras diferentes indican diferencias estadísticas de la aplicación del análisis de la varianza con test LSD Fisher, $\mathrm{p}$-valor $<0,05 .{ }^{* * *} \mathrm{p}<0,001 ; * * \mathrm{p}<0,05 ;{ }^{*} \mathrm{p}>0,05$.

La levadura Ionys se destacó primeramente con respecto a la duración promedio de la fermentación produciendo fermentaciones más lentas [10], las cuales finalizaron dos o tres días después de las demás cepas
(Tabla 3). El análisis de dos vías reveló que no hubo efecto interactivo entre levadura y zona ( $\mathrm{L}$ x $\mathrm{Z}$; p>0,05) sobre ninguno de los parámetros medidos. Sin embargo, se observó un significativo efecto individual de ambos 


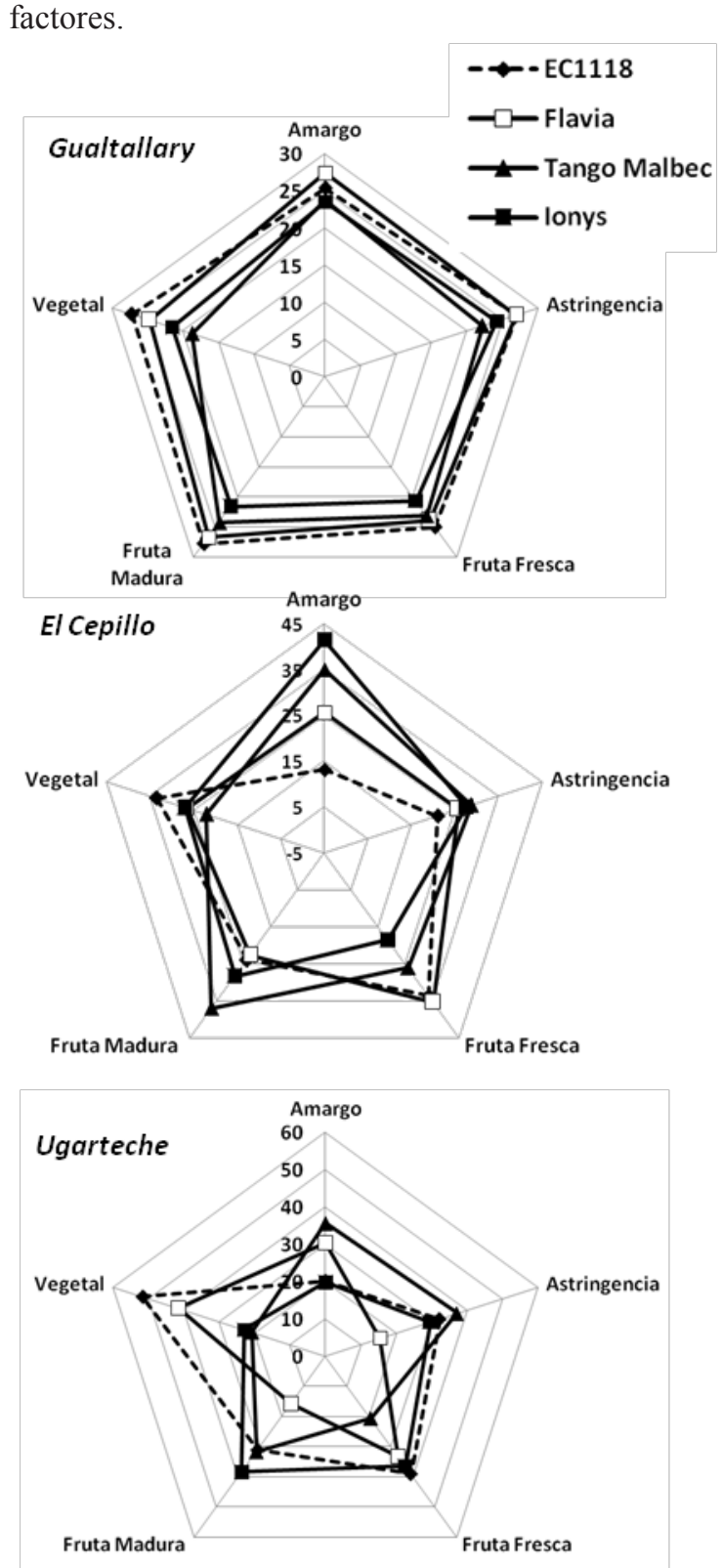

Figura 1. Media de las intensidades percibidas por los degustadores en el análisis sensorial descriptivo de los vinos Malbec de tres zonas fermentados con diferentes levaduras.

En el caso de la levadura, $\mathrm{pH}$, la acidez total y el grado alcohólico fueron fuertemente afectados por las diferentes cepas $[9,10]$. En mayor medida este efecto se observó en la cepa Ionys, y en menor medida en la cepa Flavia, las cuales produjeron un aumento de la acidez total, disminución del $\mathrm{pH}$ y del grado alcohólico. Por otro lado, debido a las diferencias detalladas en los mostos al principio de esta sección, los vinos de El Cepillo alcanzaron menores niveles de alcohol debido a su menor grado de madurez, sin embargo, los vinos de Ugarteche presentaron mayores niveles de $\mathrm{pH}$ a pesar de que sus mostos presentaban menores valores de este parámetro. Esto puede deberse al mayor contenido de potasio que poseían los mostos de Ugarteche (dato no mostrado). En cuanto a los valores de acidez volátil, Ionys produjo los menores niveles.
En la Tabla 4 se detallan las características cromáticas y de composición polifenólica determinadas en los vinos. En primer lugar, el efecto de la levadura (L) fue muy significativo $(p<0,001)$ en IC donde los mayores niveles fueron alcanzados por Ionys seguida de Tango Malbec. Este dato es interesante debido a que se sabe que la mayor intensidad colorante en el caso de Ionys es debido a su bajo $\mathrm{pH}$ [3], sin embargo no fue el caso de Tango Malbec, cuya mayor intensidad se debió al mayor contenido de antocianos libres y consecuente mayor nivel de IPT, aunque no siempre significativo, en cada zona. La levadura EC1118 alcanzó los menores niveles de estos parámetros y Flavia con un nivel un poco superior a ésta, no logró diferenciarse de la misma. En el caso del efecto producido por las zonas sobre estos parámetros se observó un efecto significativo ( $<<0,001)$, destacándose la zona de Ugarteche la cual se caracterizó por menores niveles de color, antocianos e IPT, debido principalmente sus condiciones agroecológicas, menor altitud y mayor rendimiento del viñedo. La levadura Ionys mostró en general un menor contenido de antocianos e IPT debido a que por el bajo $\mathrm{pH}$ y menor grado alcohólico de los vinos se ve favorecido el fenómeno de copigmentación eliminando del medio compuestos antocianos en forma libre y brindandole al vino mayor estabilidad en el tiempo [11].

Sensorialmente el panel de degustadores evaluó durante 3 sesiones 3 atributos aromáticos (vegetal, fruta fresca y fruta madura) y 2 atributos en boca (astringencia y amargo). El análisis estadístico no arrojó diferencias estadísticas como tampoco se observa una tipificación de las cepas en las tres zonas. Sin embargo, la levadura EC1118 se caracterizó a nivel aromático por la mayor intensidad de nota vegetal en todas las zonas (Fig. 1). Los vinos de Gualtallary, resultaron con perfiles muy similares entre sí con respecto a las levaduras, mientras que en Altamira y Ugarteche se observó un mayor efecto sensorial producido por éstas.

Con el objetivo de observar claramente la incidencia de los factores levadura y zona sobre todos los parámetros medidos, se realizó un análisis de componentes principales (ACP, Fig. 2), permitiendo explicar el $73 \%$ de la varianza existente entre los datos. En principio se observa un fuerte efecto de la zona, permitiendo una agrupación de los tratamientos según el origen de las uvas. Con respecto a la CP1, en el lado positivo de la misma se ubicaron los tratamientos correspondientes a las uvas de Gualtallary, caracterizados por la mayor intensidad de color, grado alcohólico, nota vegetal, fruta madura y astringencia. El tratamiento Ionys-G se separa con respecto a las demás levaduras acercándose a la mayor acidez total, mayor intensidad de color. Este comportamiento de Ionys se observa en las demás zonas, en las cuales se separa de las otras levaduras marcando un efecto sobre el vino, relacionándose con la nota de fruta fresca, acidez total, IPT e mayor intensidad colorante. 


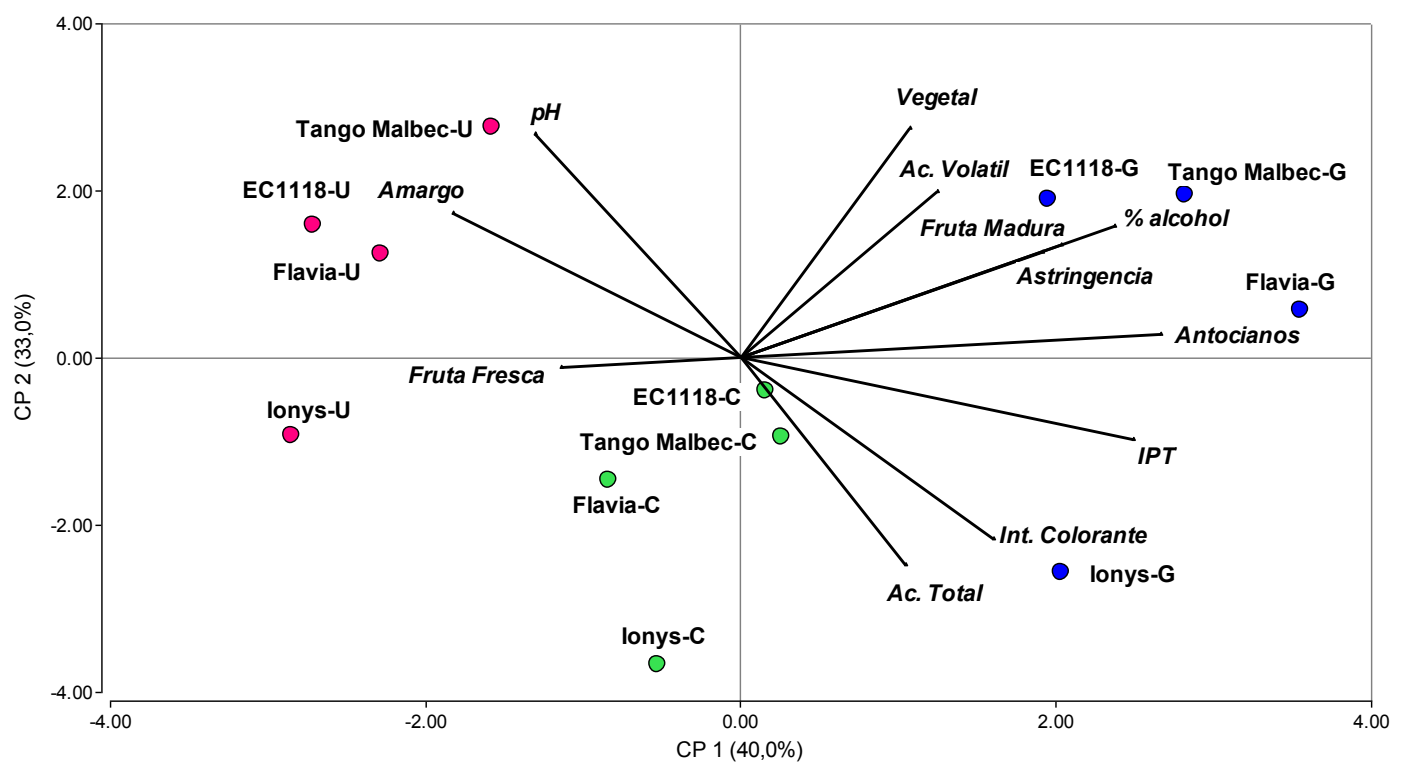

Figura 2. Análisis de componentes principales de los parámetros medidos en los vinos de la variedad Malbec provenientes de 3 regiones (C: El Cepillo, U: Ugarteche, G: Gualtallary) fermentados con 4 levaduras (EC1118, Ionys, Tango Malbec, Flavia).

\section{Conclusiones}

El objetivos del presente trabajo fue observar el efecto de las levaduras y de las zonas sobre el vino Malbec. Como conclusión, se puede afirmar que algunos aspectos del vino, principalmente los relacionados con los polifenoles, se encuentran principalmente influenciados por la región de origen de la uva (Terroir), mientras que ciertos parámetros químicos y sensoriales son determinados por la cepa de levadura utilizada.

\section{Bibliografía}

[1] DPTO. ESTADÍSTICA Y ESTUDIOS DE MERCADO SUBGERENCIA DE ESTADÍSTICA Y ASUNTOS TÉCNICOS INTERNACIONALES. Informe variedad Malbec [online]. 2017. Dostupné z: http://www.inv.gov.ar/inv_contenidos/pdf/est adisticas/tespeciales/2017/Malbec_2017.pdf

[2] CATANIA, C. a S. AVAGNINA. Malbec. In: Curso Superior de Degustación de Vinos. 2007, s. 1-11.

[3] ZAMORA, Fernando. Elaboración y crianza del vino tinto: Aspectos científicos y prácticos. Madrid: Mundi-Prensa, 2003.

[4] BERLI, F, J D'ANGELO, B CAVAGNARO, R BOTTINI, R WUILLOUD a M F SILVA. Phenolic composition in grape (Vitis vinifera $\mathrm{L}$. $\mathrm{cv}$. Malbec) ripened with different solar UV-B radiation levels by capillary zone electrophoresis. Journal of Agricultural and Food Chemistry [online]. 2008, 56(9), 2892 2898. ISSN 00218561. Dostupné z: doi:10.1021/jf073421+

[5] FANZONE, Martín. Caracterización de la composición fenólica de uvas y vinos de la variedad Malbec (Vitis vinifera L.): su relación con el origen geográfico, factores vitivinícolas y valor comercial. B.m., 2012. Universitat Rovira I Virgili, Tarragona.

[6] COSTA, Elisa, João F. DA SILVA, Fernanda COSME a António M. JORDÃO. Adaptability of some French red grape varieties cultivated at two different Portuguese terroirs: Comparative analysis with two Portuguese red grape varieties using physicochemical and phenolic parameters. Food Research International [online]. 2015, 78, 302-312. ISSN 09639969. Dostupné z: doi:10.1016/j.foodres.2015.09.029

[7] FORONI, Francesco, Miriam VIGNANDO, Marilena AIELLO, Valentina PARMA, Maurizio Guido PAOLETTI, Andrea SQUARTINI a Raffaella I. RUMIATI. The smell of terroir! Olfactory discrimination between wines of different grape variety and different terroir. Food Quality and Preference [online]. 2017, 58, 18-23. ISSN 09503293. Dostupné z: doi:10.1016/j.foodqual.2016.12.012

[8] DEL MÓNACO, Silvana M., María E. RODRÍGUEZ a Christian A. LOPES. Pichia kudriavzevii as a representative yeast of North Patagonian winemaking terroir. International Journal of Food Microbiology [online]. 2016, 230, 31-39. ISSN 18793460. Dostupné z: doi:10.1016/j.ijfoodmicro.2016.04.017

[9] TILLOY, Valentin, Anne ORTIZ-JULIEN a Sylvie DEQUIN. Reduction of Ethanol Yield and Improvement of Glycerol Formation by Adaptive Evolution of the Wine Yeast Saccharomyces cerevisiae under Hyperosmotic Conditions. Applied and Environmental Microbiology [online]. 2014, 80(8), 2623-2632. Dostupné z: doi:10.1128/AEM.03710-13

[10] ENGLEZOS, Vasileios, Francesco CRAVERO, Fabrizio TORCHIO, Kalliopi RANTSIOU, Anne ORTIZ-JULIEN, Milena LAMBRI, Vincenzo GERBI, Luca ROLLE a Luca 
COCOLIN. Oxygen availability and strain combination modulate yeast growth dynamics in mixed culture fermentations of grape must with Starmerella bacillaris and Saccharomyces cerevisiae. Food Microbiology [online]. 2018, 69, 179-188. ISSN 0740-0020. Dostupné z: doi:10.1016/j.fm.2017.08.007

[11] BOULTON, Roger B. the Copigmentation of Anthocynins and Its Role in the Color of Red Wine: Comments on a Critical Review. American Journal of Enology and Viticulture. 2001, 52(2), 67-87. ISSN 00029254.

[12] BAYONOVE, C., R. BAUMES, J. CROUZET a Z. GÜNATA. Aromas. In: A. MADRID VICENTE, ed. Enología : fundamentos científicos y tecnológicos / coord. por Claude Flanzy. $2^{\circ}$ Ed. Madrid: Mundiprensa, 2003, s. $137-176$.

[13] ROBINSON, Anthony L., Paul K. BOSS, Peter S. SOLOMON, Robert D. TRENGOVE, Hildegarde HEYMANN a Susan E. EBELER. Origins of grape and wine aroma. Part 1. Chemical components and viticultural impacts. American Journal of Enology and Viticulture. 2014, 65, 1-24.

[14] HERNÁNDEZ-ORTE, P., M. CERSOSIMO, N. LOSCOS, J. CACHO, E. GARCIAMORUNO a V. FERREIRA. The development of varietal aroma from non-floral grapes by yeasts of different genera. Food Chemistry. 2008, 107(3), 1064-1077.

[15] PÉREZ, Dolores. Efecto de la temperatura de fermentación y de la nutrición con amonio sobre la cinética de fermentación y el perfil aromático de vinos $\mathrm{cv}$. Torrontés Riojano. Mendoza, Argentina, 2016. Facultad de Ciencias Agrarias, UNCuyo.

[16] GAMERO, A. Study of the production and release of aromas during winemaking carried out by different Saccharomyces species and hybrids. Valencia, España, 2011. Universidad Politécnica de Valencia.

[17] OIV. Compendium of international methods of wine and must analysis. Vol. 1. Paris: OIV, 2013.

[18] OIV. Compendium of international methods of wine and must analysis. Vol. 2. Paris: OIV, 2013.

[19] MERCADO, L., A. DALCERO, R. MASUELLI a M. COMBINA. Diversity of Saccharomyces strains on grapes and winery surfaces: Analysis of their contribution to fermentative flora of Malbec wine from Mendoza (Argentina) during two consecutive years. Food Microbiology [online]. 2007, 24(4), 403-412. ISSN 07400020. Dostupné z: doi:10.1016/j.fm.2006.06.005 